\title{
Nuclear Magnetic Resonance Studies of the Solvation Structures of a High-Performance Nonaqueous Redox Flow Electrolyte
}

\author{
Xuchu Deng ${ }^{\mathrm{ab}}$, Mary Hu ${ }^{\mathrm{b}}$, Xiaoliang Wei ${ }^{\mathrm{b}}$, Wei Wang ${ }^{\mathrm{b}}$, Karl T. Mueller ${ }^{\mathrm{bc}}$, Zhong Chen ${ }^{\mathrm{a} *}$, Jian \\ Zhi Hu ${ }^{\mathrm{b} *}$ \\ ${ }^{a}$ Department of Electronic Science, Xiamen University, Xiamen 361005, China \\ ${ }^{\mathrm{b}}$ The Joint Center for Energy Storage Research (JCESR), \\ Pacific Northwest National Laboratory, Richland, WA 99352, USA \\ ${ }^{\mathrm{c}}$ Department of Chemistry, Penn State University, University Park, PA 16802, USA
}

\footnotetext{
* To whom correspondence should be addressed:

Jian Zhi Hu; Email: Jianzhi.Hu@pnnl.gov; Phone: (509) 371-6544; Fax: (509) 371-6546

Zhong Chen; Email: chenz@xmu.edu.cn; Phone: (+86)0592-2181712
} 
Key words: Electrolyte, Nuclear Magnetic Resonance, nonaqueous redox flow battery, solvation structures, molecular dynamics 


\section{Introduction}

As a promising stationary energy storage technology, redox flow batteries (RFBs) have experienced a recent renaissance fueled by the rapidly evolving global energy profiles because of the growing deployment of renewable energy resources. The cell design of energy-bearing liquid electrolyte stored outside the electrodes offer significant advantages including the decoupling of energy and power, excellent scalability, modular manufacturing, active thermal management, intrinsic safety, etc.[1-4] Benefiting from wider electrochemically stable voltage windows, the emerging nonaqueous RFB (NRFB) holds great potential for overcoming the low energy density challenge present in current state-of-the-art aqueous RFB technologies.[3-6] Recent research on NRFBs focuses on the development of redox active materials including organometallic compounds,[7-9] redox organic materials,[10-12] redox active polymers,[13] and the synergy between flow battery and Li-ion or Li-metal battery chemistries.[14-20] However, the current performance of NRFBs has lagged far behind their inherent capability. To date, most of them have failed to demonstrate energy density and cycling durability even close to their aqueous counterparts.

One of the major technical hurdles for NRFBs is the low solubility of redox materials leading to limited energy density.[7, 8, 12] Often, low solubility originates from insufficient solvation interactions occurring between the redox materials and solvent molecules. A good understanding of the solvation phenomena is able to offer insights to questions such as preferred functionalities and chemical sites of interactions, solvation structure evolution upon concentration changes, effects of solvation structure on material properties, etc. According to such information obtained, one can rationally design the redox molecules, improve the supporting electrolyte systems, and 
tune the flow cell conditions as redox material concentration increases. Especially, deliberate molecular engineering strategies to increase material solubility can advance the development of high energy density NRFB systems. In solvation studies, nuclear magnetic resonance (NMR) and computational modeling have proven to be powerful tools.

Recently, Wei et al. studied a new active redox species with high solubility in nonaqueous electrolytes by functionalizing pristine ferrocene $(\mathrm{Fc})$ to embody an ionically charged tetraalkylammonium (TAA) pendant arm with a bis(trifluoromethanesulfonyl)imide (TFSI) counter anion (hence denoted as Fc1N112-TFSI).[6] This compound was electrochemically tested in a Li/organic redox flow battery configuration with a cell potential of $\sim 3.5 \mathrm{~V}$. Previous work verified that the ionic pendant intensifies interactions between Fc1N112-TFSI and the mixed solvents composed of ethylene carbonate (EC), propylene carbonate (PC) and ethyl methyl carbonate (EMC), demonstrated by proton NMR experiments and density function theory (DFT) calculations.[6] Chart 1 shows a representation of the structures of all major components in this system. The major hypothesis is that the solute-solvent interactions take place predominantly at the ionic pendant arm of Fc1N112-TFSI with fully dissociated $\mathrm{TAA}^{+}$and $\mathrm{TFSI}^{-}$at Fc1N112-TFSI concentrations ranging from $0.1 \mathrm{M}$ to $1.7 \mathrm{M}$ in the solvent. However, with ${ }^{1} \mathrm{H}$ NMR alone, the complicated details of the solvation structures have not been sufficiently decoded. Additional studies utilizing Pulsed Field Gradient (PFG) NMR measurements of diffusion[21] coupled with molecular dynamics simulations argue that the PC solvent interacts with $\mathrm{Fc} 1 \mathrm{~N} 112^{+}$strongly relative to the other solvent molecule, while ${ }^{1} \mathrm{H} \mathrm{T}_{1}$ relaxation time measurements[22] probe the evolution of solvent-solvent, ion-solvent and ion-ion interactions 
during this preferential solvation. More theoretical and experimental evidence is still needed to gain comprehensive understandings of the solvation mechanism.

Here, we continue this study through using natural abundance ${ }^{13} \mathrm{C}$ and ${ }^{17} \mathrm{O}$ solution-state NMR to acquire a more accurate molecular view of the solvation structures as reported by the chemical shift values or linewidths of these two nuclides. Both, ${ }^{13} \mathrm{C}$ and ${ }^{17} \mathrm{O}$ NMR have played important roles in the development of liquid electrolytes as these methods reveal more in-depth structural details when combined with ${ }^{1} \mathrm{H}$ NMR.[23-25] Quantum chemical calculations of ${ }^{1} \mathrm{H},{ }^{13} \mathrm{C}$, and ${ }^{17} \mathrm{O}$ NMR chemical shifts using a variety of solvation models are carried out to complementarily interpret the experimental results and observations related to the detailed molecular interactions.

\section{Experimental}

\subsection{Materials and Sample Preparations}

The preparation of Fc1N112-TFSI is reported in detail elsewhere.[6, 26] Briefly, Fc1N112-TFSI was synthesized in two steps: (dimethylaminomethyl)ferrocene (96\%, Sigma-Aldrich) reacted with bromoethane (98\%, Sigma-Aldrich) in anhydrous acetonitrile (99.8\%, Sigma-Aldrich) to produce the intermediate of ferrocenylmethyl ethyl dimethylammonium bromide, followed by anion exchange with lithium bis(trifluoromethanesulfonyl)imide (LiTFSI, BASF) in deionized water to afford the Fc1N112-TFSI in an overall yield of 91\%. The NMR sample solutions were prepared simply by mixing the Fc1N112-TFSI and the solvents at specified concentrations in an argon-filled glove box (Mbraun, Stratham, NH) with moisture and oxygen levels less than 1 ppm. Battery grade PC, EC and EMC were purchased from BASF. Since EC is in solid form at room temperature, NMR spectra for neat EC were acquired by dissolving EC in deuterated 
chloroform $\left(\mathrm{CDCl}_{3}\right)$.

\subsection{NMR Measurements}

All ${ }^{13} \mathrm{C}$ NMR experiments were carried out on a Varian $600 \mathrm{MHz}$ NMR spectrometer equipped with a $\mathrm{Z}$ axis gradient $5 \mathrm{~mm}$ triple resonance probe. The corresponding Larmor frequency was 150.933 MHz. A single $90^{\circ}$ hard pulse with gated decoupling during data acquisition period was used. About 512 to 2048 scans were acquired for each spectrum with an acquisition time of about $0.9 \mathrm{~s}$ and a recycle delay time of $5 \mathrm{~s} .{ }^{13} \mathrm{C}$ NMR spectra were externally referenced to TMS (assigned a value of $0.0 \mathrm{ppm}$ ). The peak assignments for the ${ }^{13} \mathrm{C}$ NMR spectra of EC, PC, EMC and TFSI were according to those in computer databases, i.e., ACD/NMR Databases from ACDLabs (http://www.acdlabs.com). Natural abundance ${ }^{17} \mathrm{O}$ NMR experiments were performed on a Varian-Agilent $900 \mathrm{MHz}$ NMR spectrometer equipped with a home-built large-samplevolume probe $(15 \mathrm{~mm}$ outer diameter). Details of the probe design and performance will be published in a separate paper.[25] The corresponding Larmor frequency was $122.041 \mathrm{MHz}$. A 25 $\mu$ s long pulse was used for spectral excitation. The spectra were collected in 1000 - 30000 scans depending on the linewidth of the peaks, with an acquisition time of $25 \mathrm{~ms}$ and a recycle delay time of $0.5 \mathrm{~s} .{ }^{17} \mathrm{O}$ NMR spectra were referenced to the oxygen resonance from deuterium oxide $\left(\mathrm{D}_{2} \mathrm{O}\right.$, assigned a value of $\left.0.0 \mathrm{ppm}\right)$. Chemical shifts and linewidths of NMR signals were obtained by fitting Lorentzian functions to the experimental spectra using the NUTs program (v.2012, Acorn NMR Inc., Las Positas, CA, USA). All NMR measurements were carried out at room temperature $\left(20^{\circ} \mathrm{C}\right)$.

\subsection{DFT Calculations}


Quantum chemical calculations were carried out using the Gaussian09 software suite.[27] The structures of all the molecules were built using GaussView 5.[28] Geometry optimization was computed using DFT and B3LPY methods with 6-31G(d,p) basis set.[29] NMR calculations were performed based on the geometry optimized structures at the same level of the theory and with the basis set of $6-311+G(2 d, p)$ to calculate the chemical shielding for each atom except Fe, where the basis set of Fe atom is still 6-31G(d,p). The solvation mode is employed with the key word "SCRF" in the Gaussian input script, which requests that a calculation be performed in the presence of a solvent by placing the solute in a cavity within the solvent reaction field. The radius of molecules are obtained by using optimized single molecules with the key word "volume" into input script.

\section{Results and discussions}

\subsection{NMR Experiments}

\subsection{1. ${ }^{13} \mathrm{C}$ NMR Studies}

Carbon atoms are abundant in both solute and solvent molecules, especially in the Fc1N112 cation that contains 15 carbon atoms (see Chart 1), leading to high complexity in the ${ }^{13} \mathrm{C}$ NMR spectra of these samples. To help assign the peaks in mixed systems, ${ }^{13} \mathrm{C}$ NMR experiments were conducted separately on each of the solvents and their mixture, showing well distinguishable peaks (Figure S1). It is noted that the ${ }^{13} \mathrm{C}$ NMR peak positions of individual neat solvents remain unchanged in the mixtures, indicating no noticeable interactions taking place among or between these solvent molecules that are different from the interactions experienced in neat solvents. The PFG NMR results [21] on a mixture of the solvents demonstrate that the three components of the ternary solvent mixture (without solute) are equal, probably due to the common interactions 
through the carbonyl groups found in each solvent molecule, and that they lie between the measured diffusion coefficients of neat PC and neat EMC.

An expanded view of the ${ }^{13} \mathrm{C}$ NMR spectra of both pristine ferrocene and Fc1N112-TFSI dissolved in a solvent mixture of EC/PC/EMC (4/1/5 by weight) at different concentrations is shown in Figure 1 (the full spectra are plotted in Figure S2). All of the peaks are well separated and identified, shown by the numbering in the molecular structures and the spectra. Chemically equivalent carbon atoms are labeled with the same numbering, for example $\mathrm{C} 3, \mathrm{C} 6, \mathrm{C} 7$, and $\mathrm{C} 8$. The integrated peak intensity increases in accordance with the number of carbon atoms, i.e., $\mathrm{C} 1$ : C2 : C3 : C4 : C5 : C6 : C7 : C8 being $1: 1: 2: 1: 1: 2: 2: 5$, further validating our peak assignments.

Comparing the ${ }^{13} \mathrm{C}$ NMR of the solvent mixture (Figure 1a) and the solutions (Figure 1b-e), the changing of the peak positions for all solvents, if there is any, is no more than $0.22 \mathrm{ppm}$ (See Table 1 for details). Such shifts are typically negligible for ${ }^{13} \mathrm{C}$ NMR, indicating no chemical bonds formed on the solvent molecules with any solute molecules in good agreement with ${ }^{1} \mathrm{H}$ NMR studies performed previously.[6] This is further supported by the observation that the peak positions of the Fc1N112 ${ }^{+}$change slightly (by no more than $0.28 \mathrm{ppm}$ ) at the Fc1N112-TFSI concentrations from 0.1 M to saturation (Table S1). However, non-chemical interactions between the solvent and the Fc1N112-TFSI molecules are indeed occurring especially at higher Fc1N112TFSI concentrations, evidenced by the small downfield displacements (to higher ppm, dotted lines in Figure 1), for example, C1, C2, C7, and $\mathrm{C} 8$ for $\mathrm{Fc} 1 \mathrm{~N} 112^{+}$; $\mathrm{C} 3$ and $\mathrm{C} 4$ for both $\mathrm{PC}$ and EMC. Meanwhile, chemical shifts of C3, C4 and C5 also change considerably, but they change 
first upfield and then downfield with the concentrations changing from $0.1 \mathrm{M}$ to $<1.7 \mathrm{M}$ and then saturation. These changes may be related to the solvation structures changes demonstrated in ${ }^{1} \mathrm{H}$ $\mathrm{T}_{1}$ relaxation studies.[22] Fast molecular exchanges arise between bulk solvent molecules and those in solvation shells, where the exchange frequency is beyond the NMR detection time scale, leading to an averaged peak position for each carbon atoms in NMR.[25] As the Fc1N112-TFSI concentration increases, more solvent molecules will stay out of the bulk and remain for more time in the solvation shell, causing a departure of chemical shifts from their original positions for both solvents and Fc1N112 .

\subsection{2. ${ }^{17}$ O NMR Studies}

For national abundance ${ }^{17} \mathrm{O}$ NMR, the line-width matters more significantly than changes in chemical shift values. Figure 2 shows the ${ }^{17} \mathrm{O}$ NMR spectra of the Fc1N112-TFSI solutions in the same solvent mixture of EC/PC/EMC at concentrations of $0 \mathrm{M}, 0.25 \mathrm{M}, 0.85 \mathrm{M}$ and $1.70 \mathrm{M}$, respectively. The chemical shifts of all peaks remain unchanged (Table S2), which again indicates that no chemical bonds formed between the Fc1N112-TFSI and the solvent molecules. The line-width of all peaks of both solvents and $\mathrm{TFSI}^{-}$anions increase substantially with the Fc1N112-TFSI concentration, reflecting more restricted molecular motions caused by stronger solute-solvent interactions at higher concentrations.

Similar to the solvent molecules, fast exchange of the $\mathrm{TFSI}^{-}$anions also takes place between the bulk solvent and the solvation shell. When the concentration of Fc1N112-TFSI is low, i.e. 0.25 M, only a small proportion of the solvent molecules occupy the solvation shell around Fc1N112 and $\mathrm{TFSI}^{-}$, along with rapid exchanges of both solvent molecules and $\mathrm{TFSI}^{-}$anions between 
positions in the solvation shell and in the bulk. Thus most of solvent molecules are in the bulk solvent while $\mathrm{TFSI}^{-}$anions have less opportunity to stay into solvation shells of Fc1N112 ions, leading to almost unchanged peak line-width. At higher Fc1N112-TFSI concentrations, the solvent molecules are depleted from the bulk and take part in the solvation shells. Both solvents and TFSI in the solvation shells are less isotropic than those in bulk solvent due to interactions with the Fc1N112 ${ }^{+}$cations. The exchanges slow substantially, increasing the line-widths of the ${ }^{17} \mathrm{O}$ NMR peaks. A combination of ${ }^{1} \mathrm{H} \mathrm{T}_{1}$ measurements and molecular dynamics simulations provide additional molecular level details.[22]

\subsection{DFT Calculation}

The main goal of the computational studies of the NMR chemical shifts is to gain further insight into solvation phenomenon of electrolytes based on NMR experimental results. Since we employ TMS as the experimental reference standard, NMR parameters for the TMS molecule are calculated first to reference calculated results of ${ }^{1} \mathrm{H}$ and ${ }^{13} \mathrm{C}$ NMR. The absolute ${ }^{13} \mathrm{C}$ chemical shift of TMS molecule is obtained as $-182.74 \mathrm{ppm}$ by averaging shifts of four equivalent carbon atoms. Then all the other calculated results are referenced by adding $182.74 \mathrm{ppm}$ to absolute values. Similarly, calculated results of ${ }^{1} \mathrm{H}$ NMR are referenced by adding $31.86 \mathrm{ppm}$ to absolute values.

Chemical shifts of ${ }^{13} \mathrm{C}$ associated with neat EC, $\mathrm{PC}$, and EMC are calculated in the gas phase, i.e., using a single molecule. Figure 3a-c shows optimized structures of EC, PC, and EMC molecules. Comparison chemical shifts between calculated and experimental results are summarized in 
Table 1 using TMS as the reference as discussed above. Results of calculations are observed to be reasonable in agreement with experimental data.

For Fc1N112-TFSI molecule, models of Fc1N112 ${ }^{+}$ion and TFSI $^{-}$anion are developed separately. Optimized structures in the gas phase are shown in Figure 3d-e. The structure of Fc1N112+ ion agrees with previous results carried out using the ADF program.[6] Calculated chemical shifts are summarized in Table S3, where values of C3, C6, C7, and C8 represent average shifts of those atoms denoted with the same labels in Chart 1.

In order to simulate the interaction between solute and solvent, we employ the solvation mode. Both Fc1N112 ${ }^{+}$ions and Fc1N112-TFSI molecules are optimized in this mode by setting relative dielectric constants $(\varepsilon)$ and molecular radius of solvent molecules, where $\varepsilon$ of the mixed solvent is estimated by linear combination according to the molar ratio of EC/PC/EMC. Chemical shifts of ${ }^{13} \mathrm{C}$ are calculated based on the optimized structures and are summarized in Table S3. With the addition of TFSI ${ }^{-}$anion, chemical shifts of all carbon atoms of Fc1N112 undergo varying degrees of changes. In all systems, $\mathrm{C} 1$ experience downfield displacements (towards higher ppm) through the effect of TFSI. C7 and C8 shift to downfield only in environments of alkyl carbonyl solvent, i.e. in PC or the mixture of EC/PC/EMC. Detailed comparisons between experiments and calculations are organized in Table 2. In experiments, chemical shifts of saturated electrolyte are higher than those of electrolyte at concentrations of $0.1 \mathrm{M}$. In calculations, chemical shifts of TFSI-coordinated Fc1N112 are larger than those of isolated Fc1N112. The data in "shifts" row represents differences between that in row tow and row one, indicating that the shift direction of calculation agrees satisfactorily with experiments where concentrations of the solute increase. 
Therefore, isolated $\mathrm{Fc} 1 \mathrm{~N} 112^{+}$ions represent the majority form in electrolytes at low concentrations, i.e. $0.1 \mathrm{M}$. Conversely, TFSI-coordinated $\mathrm{Fc} 1 \mathrm{~N} 112^{+}$ions (i.e. contacted ion-pairs) are accounted as the greater part in electrolytes at high concentrations (i.e. saturation). The combination of experiments and calculations demonstrates that $\mathrm{Fc} 1 \mathrm{~N} 112^{+}$and $\mathrm{TFSI}^{-}$experience more opportunities to associate with each other at high concentrations. In other words, most Fc1N112-TFSI is dissociated in the alkyl carbonyl solvent at low concentrations.

In terms of ion-pair structure, Fc1N112-TFSI molecule shows slight changes with different environmental parameters. Figure 4a shows the optimized structure of Fc1N112-TFSI in the gas phase. The positive charge is distributed near the nitrogen atom of Fc1N $112^{+},[6]$ while the negative charge is concentrated on the nitrogen atom of TFSI- Consequently, the attractive force emerges between these two nitrogen atoms. The distance (D) between these two atoms is one simple measure of the extent of interaction between $\mathrm{Fc}_{\mathrm{N}} 112^{+}$and $\mathrm{TFSI}^{-}$. The structure of Fc1N112-TFSI remains unchanged by visual observation when it is placed in the solvation environment. It is worth noting that $\mathrm{D}$ is altered with variations of environmental parameters. Figure $4 \mathrm{~b}$ shows the relationship between $\mathrm{D}$ and dielectric constant $(\varepsilon)$ of solvent, where $\varepsilon$ of the gas phase is equivalent to 1 . The data can be fit with a curve described by an inverted exponential form. The change of D indicates that TFSI ${ }^{-}$moves away from $\mathrm{Fc} 1 \mathrm{~N} 112^{+}$with increased value of $\varepsilon$. In other words, it is more probable for the solvent with high $\varepsilon$ to dissociate the Fc1N112-TFSI ion pair.

In addition, we have investigated the effect of solute molecules on solvent molecules. In the NMR experiments, ${ }^{13} \mathrm{C}$ chemical shifts of PC and EMC experience minor changes with 
concentrations of Fc1N112 ${ }^{+}$. For the calculations, we consider two cases of solute molecule, i.e. dissociation and contact. In the case of dissociated ion-pair, single solvent molecules (i.e. PC and EMC) are placed together with Fc1N112 ${ }^{+}$ion and TFSI $^{-}$anion respectively (Figure 5a-b). After optimization, carbonyl oxygen $\left(\mathrm{O}_{\mathrm{C}}\right)$ atoms orient towards the nitrogen atom of Fc1N112, indicating that cations are coordinated by $\mathrm{O}_{C}$ atoms of solvent molecules. The $\mathrm{O}_{\mathrm{C}}$ is attracted by the pendant arm due to the positive charge on the nitrogen atom and lone ion pair of $\mathrm{O}_{\mathrm{C}}$. Based on the optimization, calculated chemical shifts of ${ }^{13} \mathrm{C}$ of PC and EMC are summarized in Table 3. Chemical shift values of majorities of carbon atoms increase (shift downfield) when solvent molecules are coordinated with either Fc1N112 ${ }^{+}$or $\mathrm{TFSI}^{-}$. Although the chemical shift of $\mathrm{C} 4$ of PC becomes slightly smaller due to the association with of $\mathrm{Fc} 1 \mathrm{~N} 112^{+}$, the interaction of TFSI drives it towards the opposite direction more severely. Since the ratio of Fc1N112 ${ }^{+}$to TFSI $^{-}$in electrolytes is 1:1, the total effect of Fc1N112-TFSI on the chemical shifts can be estimated in accordance with the following equation.

$$
\delta_{E}=\left(\delta_{F}-\delta_{S}\right)+\left(\delta_{T}-\delta_{S}\right)
$$

where $\delta_{S}, \delta_{F}$, and $\delta_{T}$ are chemical shifts of single, Fc1N112-coordinated, and TFSI-coordinated molecules, respectively. For $\mathrm{C} 4$ of $\mathrm{PC}, \delta_{S}=19.47 \mathrm{ppm}, \delta_{F}=19.30 \mathrm{ppm}, \delta_{T}=20.50 \mathrm{ppm}$, thus the estimated total displacement, $\delta_{E}$, is calculated to be $0.86 \mathrm{ppm}$. Similarly, C3 and C4 of EMC receive combined effects of interactions with solute molecules, and then shifts of $0.6 \mathrm{ppm}$ and $0.52 \mathrm{ppm}$ are predicted based on Eq. [1]. In general, carbon atoms of both PC and EMC become less shielding (shift towards higher ppm), when PC and EMC interact with solute molecules, or when solvent molecules form solvation shells. In the case of contact ion-pair, we develop a system of solvent molecule coordinated by contacted Fc1N112-TFSI pair. Optimized structures show that solvent molecules are located near the $\mathrm{Cp}$ ring of solute molecule (Figure 
5c). It can be observed from Table 3 that ${ }^{13} \mathrm{C}$ chemical shifts of solvent molecules in this system move downfield compared with those of single solvent molecules (except C4 of EMC).

In experiments, relatively small values for perturbations of chemical shifts arise at low concentrations, i.e. $0.1 \mathrm{M}$, while large values of shifts are measured at high concentrations, i.e. saturation (Table 1). Therefore, we conclued that most of solvent molecules are found as bulk solvent in electrolytes at low concentrations, resulting in small changes of chemical shifts. Conversely, majorities of solvent molecules are coordinated with $\mathrm{Fc}_{\mathrm{N}} 112^{+}$or $\mathrm{TFSI}^{-}$, or Fc1N112-TFSI contact ion-pairs, thus behaving as direct solvation shells in electrolytes at high concentrations, leading to larger shifts. Based on simple chemical exchange theory, spectra show average chemical shifts of in the fast exchange regime, and therefore the bulk solvent constitutes the majority species at low concentrations, and the observed average shifts approach those of mixed solvent (Figure 1a). Similarly, the bulk solvent gradually forms solvation shells with the increase of concentrations, causing the dominance of solvent molecules in solvent shells, and resulting in peak-center displacements towards those of coordinated solvent molecules.

Taking advantage of optimized structures in ${ }^{13} \mathrm{C}$ NMR calculations, we perform ${ }^{1} \mathrm{H}$ chemical shifts calculations of solvent molecules, and results are summarized in Table S4. Notably, calculated values of free solvent molecules are in disagreement with chemical shifts of mixed solvent in experiments. To account for more complex intermolecular interaction, we perform solvent molecules ${ }^{1} \mathrm{H}$ NMR calculations by using multiple copies of solvent molecules surrounding a central solvent molecule and consider only the values obtained from the central one (Figure 5d). With increases in the number of solvent molecules, ${ }^{1} \mathrm{H}$ chemical shifts move 
towards downfield. When the numbers of solvent molecules are five, i.e. one is surrounded by four, for EC, or six for PC and EMC, calculated chemical shifts of central ones are satisfactorily in agreement with those of mixed solvent in experiments (See Table 4 for comparisons).

Similar to ${ }^{13} \mathrm{C}$ calculations, ${ }^{1} \mathrm{H}$ shifts of solvent molecules in the present of Fc1N112-TFSI can be estimated by taking averages of values of Fc1N112-coordinated and TFSI-coordinated systems (Table S4). It can be observed from the table that estimated results disagree with experimental results at either low or saturated concentration. In the case of $0.1 \mathrm{M}$ concentration, number of bulk solvent molecules is far greater than that within solvation shells, thus the effect of coordinated solvent molecules on the observed ${ }^{1} \mathrm{H}$ chemical shifts is negligible under rapid molecular exchange. On the other hand, because most solvent molecules are in solvation shell in saturated electrolyte, coordinated species dominate observed chemical shifts. Disagreements of chemical shifts of solvent molecules between the ion-coordinated in calculations and saturated electrolyte in experiments indicate that $\mathrm{Fc}_{1 \mathrm{~N}} 112^{+}$and $\mathrm{TFSI}^{-}$are contacted ion-pair rather than dissociated at saturated concentration. We use structure of Fc1N112-TFSI-coordinated solvent molecules to verify the conclusion (Figure 5c). Based on optimized structures, calculated ${ }^{1} \mathrm{H}$ chemical shifts agree satisfactorily with those of saturated electrolyte in experiments (Table 4). Additionally, shifts of solvent molecules move upfield (towards small ppm values) under the effect of solute molecule, and differences between these two systems in calculations (Table 4) are in agreement with displacements from low to high concentrations in experiments (Table S5). These results confirm that major type of ion-pair in saturated electrolyte is contact rather than dissociation. This argument is further supported by electrochemical performance of the solute at different concentrations. In the cyclic voltammograms (CVs) of Fc1N112-TFSI shown in Figure 
$\mathrm{S} 3$, the oxidation and reduction peak separations at $5.0 \mathrm{mM}$ are significantly smaller than those at $0.1 \mathrm{M}$. The smaller peak separation reflects higher electrochemical reversibility under lower solute concentration. The Fc1N $112^{+}$and $\mathrm{TFSI}^{-}$ions are more dissociated, have better mobility and thus respond more rapidly to the sweeping voltage. This is in good agreement with the NMR study.

Previous work has verified that dimethyl sulfoxide (DMSO) is more suitable as a reference for ${ }^{17} \mathrm{O}$ NMR calculations than $\mathrm{H}_{2} \mathrm{O}$.[25] Additionally, the ${ }^{17} \mathrm{O}$ chemical shift of DMSO is $\sim 20 \mathrm{ppm}$ larger than that of $\mathrm{H}_{2} \mathrm{O} \cdot[25,30,31]$ Hence, calculated absolute shifts are converted to results of calculations by adding $269.51 \mathrm{ppm}$ where the absolute shift of DMSO is calculated to be 249.51 ppm.

Since intermolecular interactions in liquid phase would affect ${ }^{17} \mathrm{O}$ chemical shifts,[25] calculations on both single and double solvent molecules are carried out. Results using double molecules are in much better agreement with the values obtained from experiments (see Table S6 for detail). Using optimized structures of solvent molecules coordinated by $\mathrm{Fc} 1 \mathrm{~N} 112^{+}$or TFSI ${ }^{-}$(as discussed above), we compute ${ }^{17} \mathrm{O}$ chemical shifts of solvent molecules (Table S6). Attentive comparisons of results between calculations and experiments are organized in Table 5. Similar to ${ }^{1} \mathrm{H}$ calculations, ${ }^{17} \mathrm{O}$ shifts of solvent molecules that are influenced by Fc1N112 $2^{+}$or TFSI $^{-}$are fairly different, and those shifts under total effect of Fc1N112-TFSI contact ion-pairs can be estimated by taking averages of values of Fc1N112-coordinated and TFSI-coordinated systems (Table 5). Because of the insignificant changes of experimental ${ }^{17} \mathrm{O}$ shifts in electrolytes at different concentrations (Figure 2), we calculate average values from systems of $0.25 \mathrm{M}$, 
$0.85 \mathrm{M}$ and $1.7 \mathrm{M}$ in order to facilitate comparisons. It is observed in Table 5 that estimated results agree excellently with results of experiments. The consistency acts as a strong evidence of fast exchanges of solvent molecules. Specifically, solvent molecules in solvation shells of both Fc1N112 ${ }^{+}$and TFSI $^{-}$exchange with those in the bulk solvent, resulting in averaged ${ }^{17} \mathrm{O}$ chemical shifts.

\section{Conclusion}

We have performed ${ }^{13} \mathrm{C}$ and ${ }^{17} \mathrm{O}$ NMR to understand the dissolution mechanism of Fc1N112TFSI in mixed solvent composed of EC, PC, and EMC. The DFT calculations on ${ }^{1} \mathrm{H}$, ${ }^{13} \mathrm{C}$, and ${ }^{17} \mathrm{O}$ NMR reveal molecular-level details of chemical shifts and linewidth change with concentrations of solute. Interactions between solute and solvent molecules, ion association, and molecular motions are strongly proved by both experimental and theoretic studies. At dilute solute concentrations, the cation and the anion associated with Fc1N112-TFSI are dissociated, forming their own solvation shells. Molecules of EC, PC and EMC surround Fc1N112 ${ }^{+}$ions as solvation shells, leading to the high solubility of Fc1N112-TFSI. In this case, TFSI ${ }^{-}$anions have rare opportunities to influence chemical shifts of Fc1N112 ${ }^{+}$. Rapid exchanges of both $\mathrm{TFSI}^{-}$ anions and solvent molecules take place between solvation shells and bulk, resulting in average observed chemical shifts in NMR experiments. Molecules in the bulk solvent outnumber those in solvation shells, and dominate chemical shifts of the solvent. With increasing concentrations of solute, Fc1N112-TFSI gradually turns into solvent-shared state. At saturated concentration, contact ion pairs are formed and the solvent molecules are interacting with the Fc rings rather than interacting with the ionic pendant arm of Fc1N112-TFSI. In this case, molecular motion is 


\section{Acknowledgment}

This work was led intellectually as part of the Joint Center for Energy Storage Research (JCESR), an Energy Innovation Hub funded by the U.S. Department of Energy, Office of Science, Basic Energy Sciences (BES). The NMR sample preparations were supported by the funding from the U.S. Department of Energy's (DOE's) Office of Electricity Delivery and Energy Reliability (OE) (under Contract No. 57558). The NMR, and computational studies were conducted in the William R. Wiley Environmental Molecular Sciences Laboratory (EMSL), a national scientific user facility sponsored by DOE's Office of Biological and Environmental Research (BER) and located at PNNL. Xuchu Deng was partially supported by the National Natural Science Fund of 
China under Grant 21327001. PNNL is operated by Battelle for the Department of Energy under Contract DE-AC05- 76RLO1830. 


\section{Reference}

[1] A.Z. Weber, M.M. Mench, J.P. Meyers, P.N. Ross, J.T. Gostick, Q.H. Liu, J Appl Electrochem, 41 (2011) 1137-1164.

[2] M. Skyllas-Kazacos, M.H. Chakrabarti, S.A. Hajimolana, F.S. Mjalli, M. Saleem, J Electrochem Soc, 158 (2011) R55-R79.

[3] P. Leung, X.H. Li, C.P. de Leon, L. Berlouis, C.T.J. Low, F.C. Walsh, RSC Adv., 2 (2012) 1012510156.

[4] W. Wang, Q.T. Luo, B. Li, X.L. Wei, L.Y. Li, Z.G. Yang, Adv Funct Mater, 23 (2013) 970-986.

[5] S.H. Shin, S.H. Yun, S.H. Moon, RSC Adv., 3 (2013) 9095-9116.

[6] X.L. Wei, L. Cosimbescu, W. Xu, J.Z. Hu, M. Vijayakumar, J. Feng, M.Y. Hu, X.C. Deng, J. Xiao, J. Liu, V. Sprenkle, W. Wang, Adv. Energy Mater., 5 (2015).

[7] P.J. Cappillino, H.D. Pratt, N.S. Hudak, N.C. Tomson, T.M. Anderson, M.R. Anstey, Adv. Energy Mater., 4 (2014).

[8] A.E.S. Sleightholme, A.A. Shinkle, Q.H. Liu, Y.D. Li, C.W. Monroe, L.T. Thompson, J Power Sources, 196 (2011) 5742-5745.

[9] H.D. Pratt, A.J. Rose, C.L. Staiger, D. Ingersoll, T.M. Anderson, Dalton transactions, 40 (2011) 11396-11401.

[10] X.L. Wei, W. Xu, J.H. Huang, L. Zhang, E. Walter, C. Lawrence , M. Vijayakumar, W.A. Henderson, T.B. Liu, L. Cosimbescu, B. Li, V. Sprenkle, W. Wang, Angew Chem Int Edit, 54 (2015) $8684-8687$.

[11] F.R. Brushett, J.T. Vaughey, A.N. Jansen, Adv. Energy Mater., 2 (2012) 1390-1396.

[12] Z. Li, S. Li, S.Q. Liu, K.L. Huang, D. Fang, F.C. Wang, S. Peng, Electrochem Solid St, 14 (2011) A171-A173.

[13] G. Nagarjuna, J.S. Hui, K.J. Cheng, T. Lichtenstein, M. Shen, J.S. Moore, J. Rodriguez-Lopez, J Am Chem Soc, 136 (2014) 16309-16316. 
[14] F.Y. Fan, W.H. Woodford, Z. Li, N. Baram, K.C. Smith, A. Helal, G.H. McKinley, W.C. Carter, Y.M. Chiang, Nano Lett, 14 (2014) 2210-2218.

[15] Y. Yang, G.Y. Zheng, Y. Cui, Energy Environ. Sci., 6 (2013) 1552-1558.

[16] S. Hamelet, D. Larcher, L. Dupont, J.M. Tarascon, J Electrochem Soc, 160 (2013) A516-A520.

[17] Y.R. Wang, P. He, H.S. Zhou, Adv. Energy Mater., 2 (2012) 770-779.

[18] W. Wang, W. Xu, L. Cosimbescu, D.W. Choi, L.Y. Li, Z.G. Yang, Chem Commun, 48 (2012) 66696671.

[19] M. Duduta, B. Ho, V.C. Wood, P. Limthongkul, V.E. Brunini, W.C. Carter, Y.M. Chiang, Adv. Energy Mater., 1 (2011) 511-516.

[20] X. Wei, W. Xu, M. Vijayakumar, L. Cosimbescu, L. Tianbiao, V. Sprenkle, W. Wang, Adv Mater, 26 (2014) 7649-7653.

[21] K.S. Han, N.N. Rajput, X.L. Wei, W. Wang, J.Z. Hu, K.A. Persson, K.T. Mueller, J Chem Phys, 141 (2014).

[22] K.S. Han, N.N. Rajput, M. Vijayakumar, X.L. Wei, W. Wang, J.Z. Hu, K.A. Persson, K.T. Mueller, Preferential Solvation of an Asymmetric Redox Molecule. Submitted, (2015).

[23] X. Bogle, R. Vazquez, S. Greenbaum, A.V. Cresce, K. Xu, J. Phys. Chem. Lett., 4 (2013) 16641668.

[24] L. Yang, A. Xiao, B.L. Lucht, J Mol Liq, 154 (2010) 131-133.

[25] X. Deng, M.Y. Hu, X. Wei, W. Wang, Z. Chen, J. Liu, J.Z. Hu, J Power Sources, 285 (2015) 146155.

[26] L. Cosimbescu, X. Wei, M. Vijayakumar, W. Xu, M.L. Helm, S.D. Burton, C.M. Sorensen, J. Liu, V. Sprenkle, W. Wang, Sci Rep-Uk, Accepted (2015).

[27] M.J.T. Frisch, G. W.; Schlegel, H. B.; Scuseria, G. E.; Robb, M. A.; Cheeseman, J. R.; Scalmani, G.; Barone, V.; Mennucci, B.; Petersson, G. A.; Nakatsuji, H.; Caricato, M.; Li, X.; Hratchian, H. P.; Izmaylov, A. F.; Bloino, J.; Zheng, G.; Sonnenberg, J. L.; Hada, M.; Ehara, M.; Toyota, K.; Fukuda, R.; 
Hasegawa, J.; Ishida, M.; Nakajima, T.; Honda, Y.; Kitao, O.; Nakai, H.; Vreven, T.; Montgomery, J. A., Jr.; Peralta, J. E.; Ogliaro, F.; Bearpark, M.; Heyd, J. J.; Brothers, E.; Kudin, K. N.; Staroverov, V. N.; Kobayashi, R.; Normand, J.; Raghavachari, K.; Rendell, A.; Burant, J. C.; Iyengar, S. S.; Tomasi, J.;

Cossi, M.; Rega, N.; Millam, J. M.; Klene, M.; Knox, J. E.; Cross, J. B.; Bakken, V.; Adamo, C.;

Jaramillo, J.; Gomperts, R.; Stratmann, R. E.; Yazyev, O.; Austin, A. J.; Cammi, R.; Pomelli, C.;

Ochterski, J. W.; Martin, R. L.; Morokuma, K.; Zakrzewski, V. G.; Voth, G. A.; Salvador, P.;

Dannenberg, J. J.; Dapprich, S.; Daniels, A. D.; Farkas, Ö.; Foresman, J. B.; Ortiz, J. V.; Cioslowski, J.;

Fox, D. J. Gaussian, Inc., Wallingford CT, (2009).

[28] R.K. Dennington, Todd; Millam, John. Semichem Inc., Shawnee Mission, KS, (2009).

[29] A.D. Becke, Phys Rev A, 38 (1988) 3098-3100.

[30] J.C. Dyer, D.L. Harris, S.A. Evans, J Org Chem, 47 (1982) 3660-3664.

[31] E. Block, A.A. Bazzi, J.B. Lambert, S.M. Wharry, K.K. Andersen, D.C. Dittmer, B.H. Patwardhan, D.J.H. Smith, J Org Chem, 45 (1980) 4807-4810. 
Table $1 .{ }^{13} \mathrm{C}$ chemical shifts of solvent molecules in both experiments and calculations (ppm).

\begin{tabular}{|c|c|c|c|c|c|c|c|c|c|c|}
\hline \multirow[b]{2}{*}{ Systems } & \multicolumn{2}{|c|}{ EC } & \multicolumn{4}{|c|}{$\mathrm{PC}$} & \multicolumn{4}{|c|}{ EMC } \\
\hline & $\mathrm{C} 1$ & $\begin{array}{l}\mathrm{C} 2, \\
\mathrm{C} 3\end{array}$ & $\mathrm{C} 1$ & $\mathrm{C} 2$ & $\mathrm{C} 3$ & $\mathrm{C} 4$ & $\mathrm{C} 1$ & $\mathrm{C} 2$ & $\mathrm{C} 3$ & $\mathrm{C} 4$ \\
\hline EC/PC/EMC & 155.92 & 65.08 & 155.29 & 74.00 & 70.75 & 18.30 & 155.68 & 63.68 & 54.00 & 13.50 \\
\hline $\begin{array}{c}0.2 \mathrm{M} \text { pristine } \\
\mathrm{Fc}\end{array}$ & 155.91 & 65.09 & 155.29 & 74.00 & 70.76 & 18.35 & 155.69 & 63.71 & 54.04 & 13.56 \\
\hline $\begin{array}{c}0.1 \mathrm{M} \\
\text { Fc1N112-TFSI }\end{array}$ & 155.92 & 65.05 & 155.29 & 73.99 & 70.73 & 18.30 & 155.66 & 63.66 & 53.97 & 13.49 \\
\hline $\begin{array}{c}<1.7 \mathrm{M} \\
\text { Fc1N112-TFSI }\end{array}$ & 155.92 & 64.99 & 155.30 & 73.96 & 70.67 & 18.38 & 155.52 & 63.70 & 54.07 & 13.61 \\
\hline $\begin{array}{c}\text { Saturated } \\
\text { Fc1N112-TFSI }\end{array}$ & 156.03 & 65.05 & 155.41 & 74.07 & 70.78 & 18.48 & 155.63 & 63.79 & 54.18 & 13.71 \\
\hline $\begin{array}{c}\text { DFT } \\
\text { Calculations }\end{array}$ & 161.19 & 66.15 & 161.08 & 77.47 & 73.93 & 19.47 & 165.04 & 67.87 & 55.99 & 15.33 \\
\hline
\end{tabular}


Table 2. Comparisons of ${ }^{13} \mathrm{C}$ chemical shifts on Fc1N112 between experiments and calculations (ppm). ${ }^{\mathrm{a}}$

\begin{tabular}{ccccccc}
\hline \multirow{2}{*}{ Systems } & \multicolumn{2}{c}{ C1 } & \multicolumn{2}{c}{ C7 } & \multicolumn{2}{c}{ C8 } \\
\cline { 2 - 7 } & Exp. & Cal. & Exp. & Cal. & Exp. & Cal. \\
\hline Low concentration & 7.40 & 9.73 & 70.30 & 75.64 & 69.14 & 74.14 \\
High concentration & 7.49 & 9.77 & 70.46 & 76.11 & 69.30 & 74.44 \\
Shifts & 0.09 & 0.04 & 0.16 & 0.47 & 0.16 & 0.30 \\
\hline
\end{tabular}

${ }^{\mathrm{a}}$ For experimental data, low and high concentrations are corresponding to $0.1 \mathrm{M}$ and saturation respectively; for calculated data, low concentration indicates isolated $\mathrm{Fc} 1 \mathrm{~N} 112^{+}$ion in solvation mode, while high concentration indicates Fc1N112-TFSI pair in solvation mode. 
Table 3. Calculated ${ }^{13} \mathrm{C}$ chemical shifts of solvent in different systems (ppm).

\begin{tabular}{|c|c|c|c|c|c|c|c|c|}
\hline \multirow{2}{*}{ Systems } & \multicolumn{4}{|c|}{ PC } & \multicolumn{4}{|c|}{ EMC } \\
\hline & $\mathrm{C} 1$ & $\mathrm{C} 2$ & $\mathrm{C} 3$ & $\mathrm{C} 4$ & $\mathrm{C} 1$ & $\mathrm{C} 2$ & $\mathrm{C} 3$ & $\mathrm{C} 4$ \\
\hline $\begin{array}{c}\text { Single } \\
\text { molecule }\end{array}$ & 161.08 & 77.47 & 73.93 & 19.47 & 165.04 & 67.87 & 55.99 & 15.33 \\
\hline $\begin{array}{l}\text { Fc1N112- } \\
\text { coordinated }\end{array}$ & 166.12 & 82.14 & 76.27 & 19.30 & 167.97 & 70.02 & 56.82 & 15.05 \\
\hline $\begin{array}{c}\text { TFSI- } \\
\text { coordinated }\end{array}$ & 164.87 & 79.73 & 75.54 & 20.50 & 164.97 & 68.33 & 55.76 & 16.13 \\
\hline $\begin{array}{c}\text { Fc1N112- } \\
\text { TFSI- } \\
\text { coordinated }\end{array}$ & 162.50 & 78.87 & 74.48 & 19.52 & 165.83 & 69.08 & 56.37 & 15.07 \\
\hline
\end{tabular}


Table 4. Comparisons of ${ }^{1} \mathrm{H}$ chemical shifts on solvent molecules between calculations ${ }^{\mathrm{a}}$ and experiments ${ }^{\mathrm{b}}$ (ppm).

\begin{tabular}{|c|c|c|c|c|c|c|c|c|}
\hline \multirow{3}{*}{$\begin{array}{c}\text { Systems } \\
\begin{array}{c}\text { Surrounded solvent } \\
\text { molecules (Cal.) }\end{array}\end{array}$} & \multirow{3}{*}{$\begin{array}{c}\mathrm{EC} \\
\mathrm{H} 2 \\
\mathrm{H} 3 \\
4.89\end{array}$} & \multicolumn{4}{|c|}{$\mathrm{PC}$} & \multicolumn{3}{|c|}{ EMC } \\
\hline & & $\mathrm{H} 2$ & \multicolumn{2}{|c|}{$\mathrm{H} 3$} & $\mathrm{H} 4$ & $\mathrm{H} 2$ & $\mathrm{H} 3$ & $\mathrm{H} 4$ \\
\hline & & 5.33 & 4.76 & 4.25 & 1.57 & 4.61 & 3.80 & 1.33 \\
\hline EC/PC/EMC (Exp.) & 4.91 & 5.28 & 4.97 & 4.44 & 1.81 & 4.51 & 4.09 & 1.62 \\
\hline $\begin{array}{l}\text { Fc1N112-TFSI- } \\
\text { coordinated solvent } \\
\text { molecules (Cal.) }\end{array}$ & 4.45 & 4.84 & 4.44 & 3.88 & 1.41 & 4.24 & 3.80 & 1.29 \\
\hline $\begin{array}{c}\text { Saturated Fc1N112- } \\
\text { TFSI (Exp.) }\end{array}$ & 4.48 & 4.86 & 4.55 & 4.01 & 1.39 & 4.10 & 3.68 & 1.22 \\
\hline Displacements $^{\mathrm{c}}$ & -0.44 & -0.49 & -0.32 & -0.37 & -0.16 & -0.37 & 0 & -0.04 \\
\hline
\end{tabular}


Table 5. Comparisons of ${ }^{17} \mathrm{O}$ chemical shifts on solvent molecules between experiments and calculations (ppm).

\begin{tabular}{cccccc}
\hline \multirow{2}{*}{ Systems } & EC & \multicolumn{2}{c}{ PC } & \multicolumn{2}{c}{ EMC } \\
\cline { 2 - 6 } & O2, O3 & O2 & O3 & O2 & O3 \\
\hline Fc1N112-Coordinated & 112.62 & 141.17 & 120.45 & 127.39 & 98.58 \\
TFSI-Coordinated & 107.78 & 135.85 & 107.78 & 120.09 & 89.30 \\
Calculated Average $^{\text {a }}$ & 110.20 & 138.51 & 114.12 & 123.74 & 93.94 \\
Experimental Average $^{\text {a }}$ Averages of values of Fc1N112-Coordinated and TFSI-Coordinated. & &
\end{tabular}



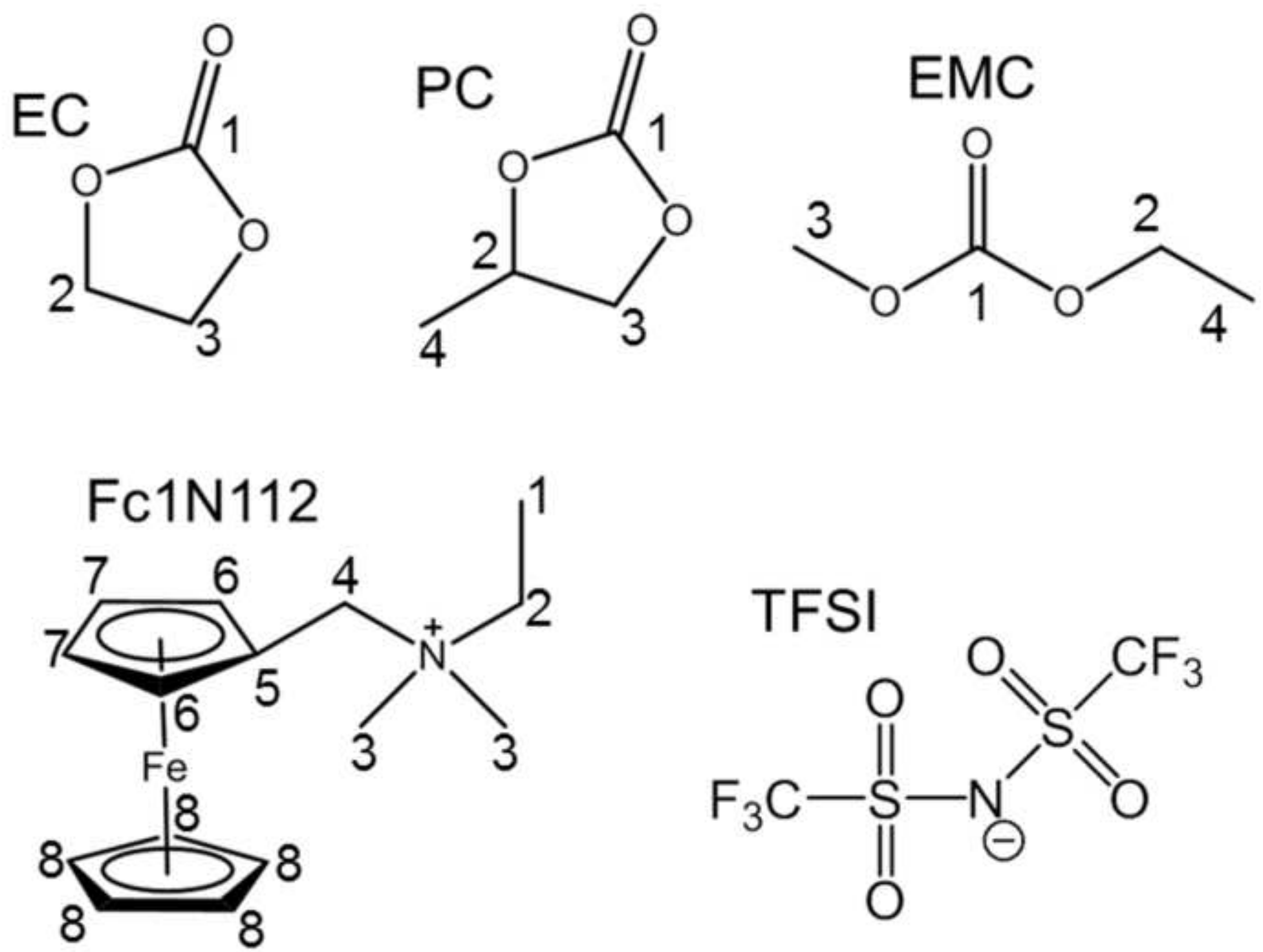


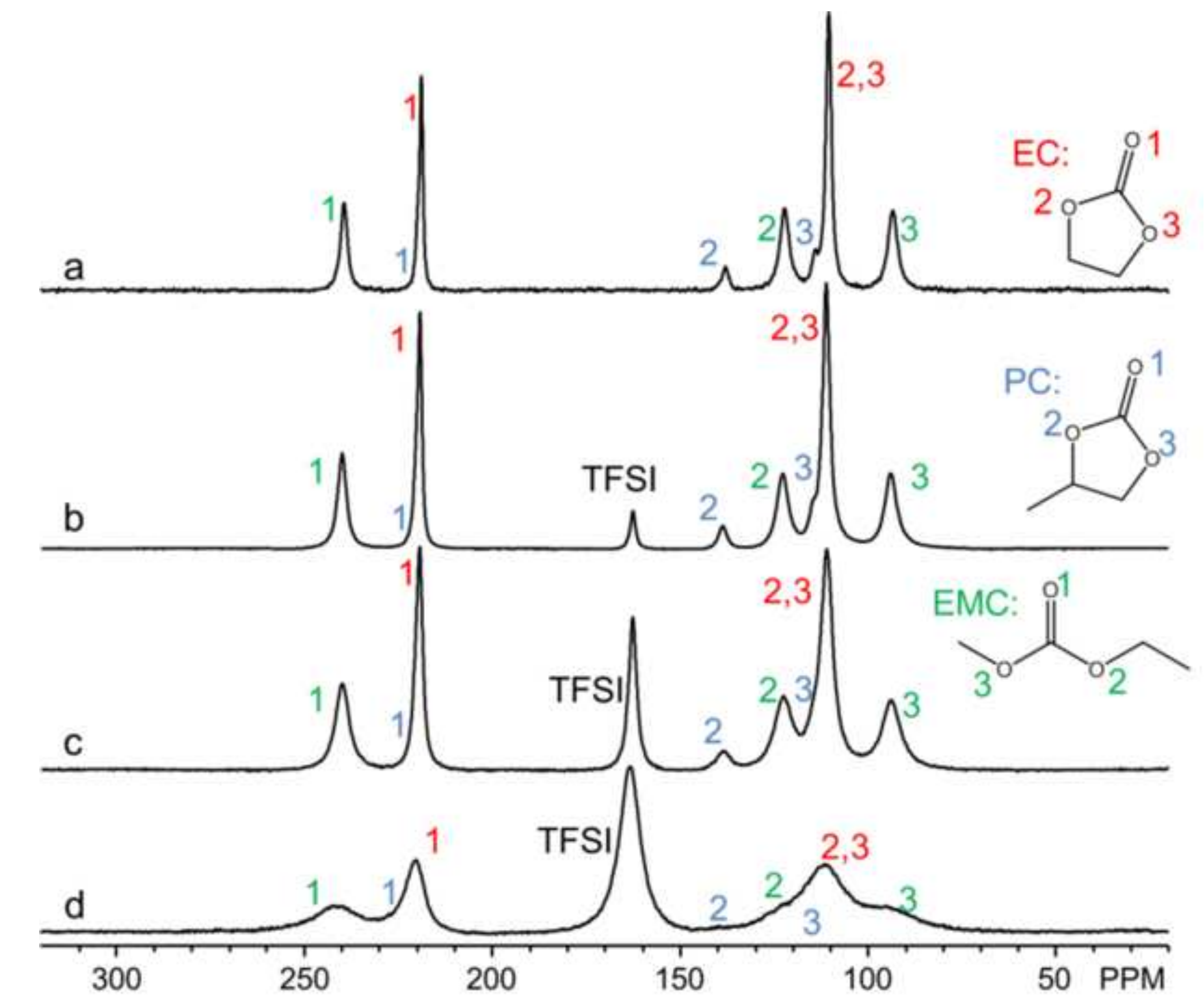



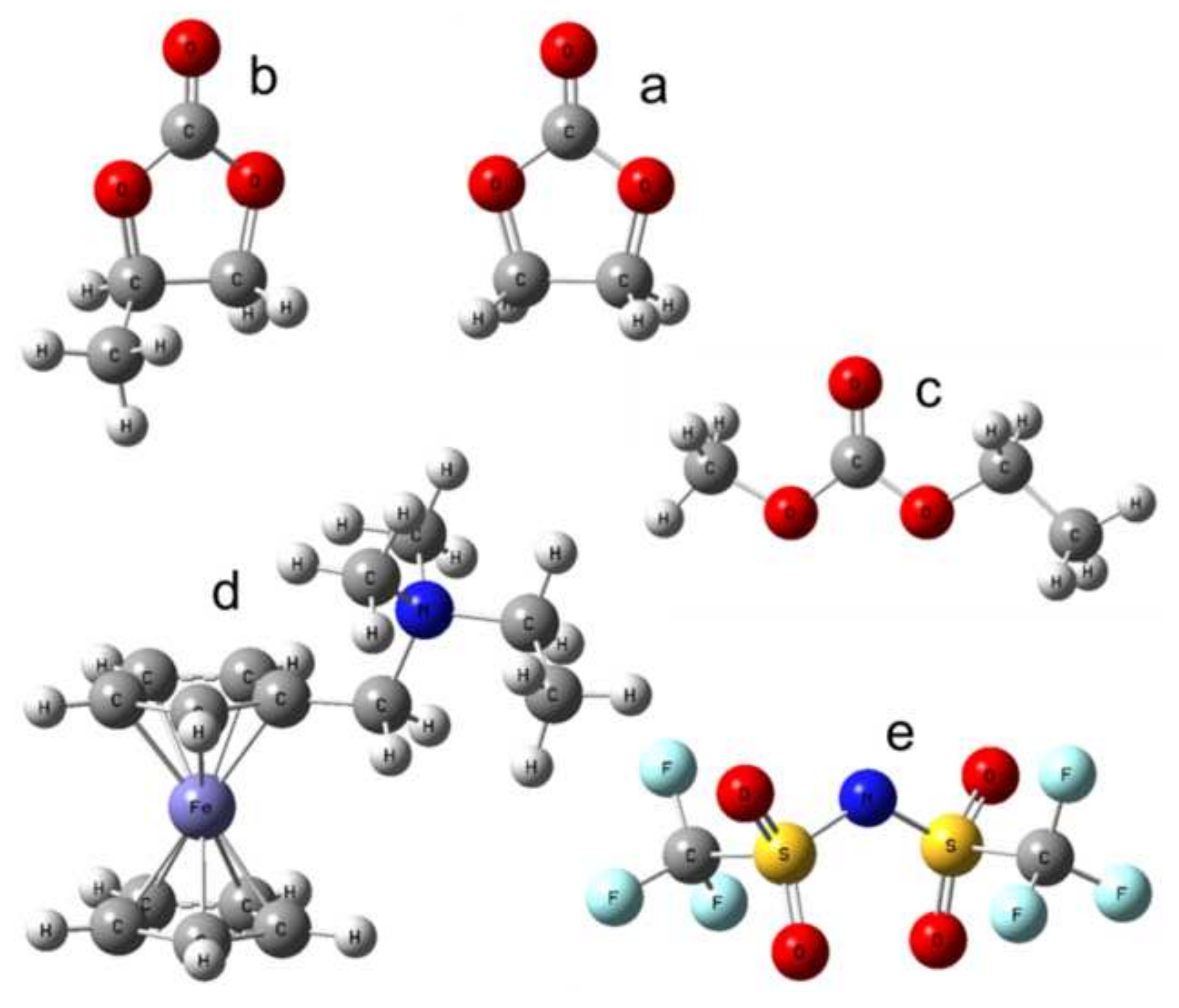

Figure 3

.



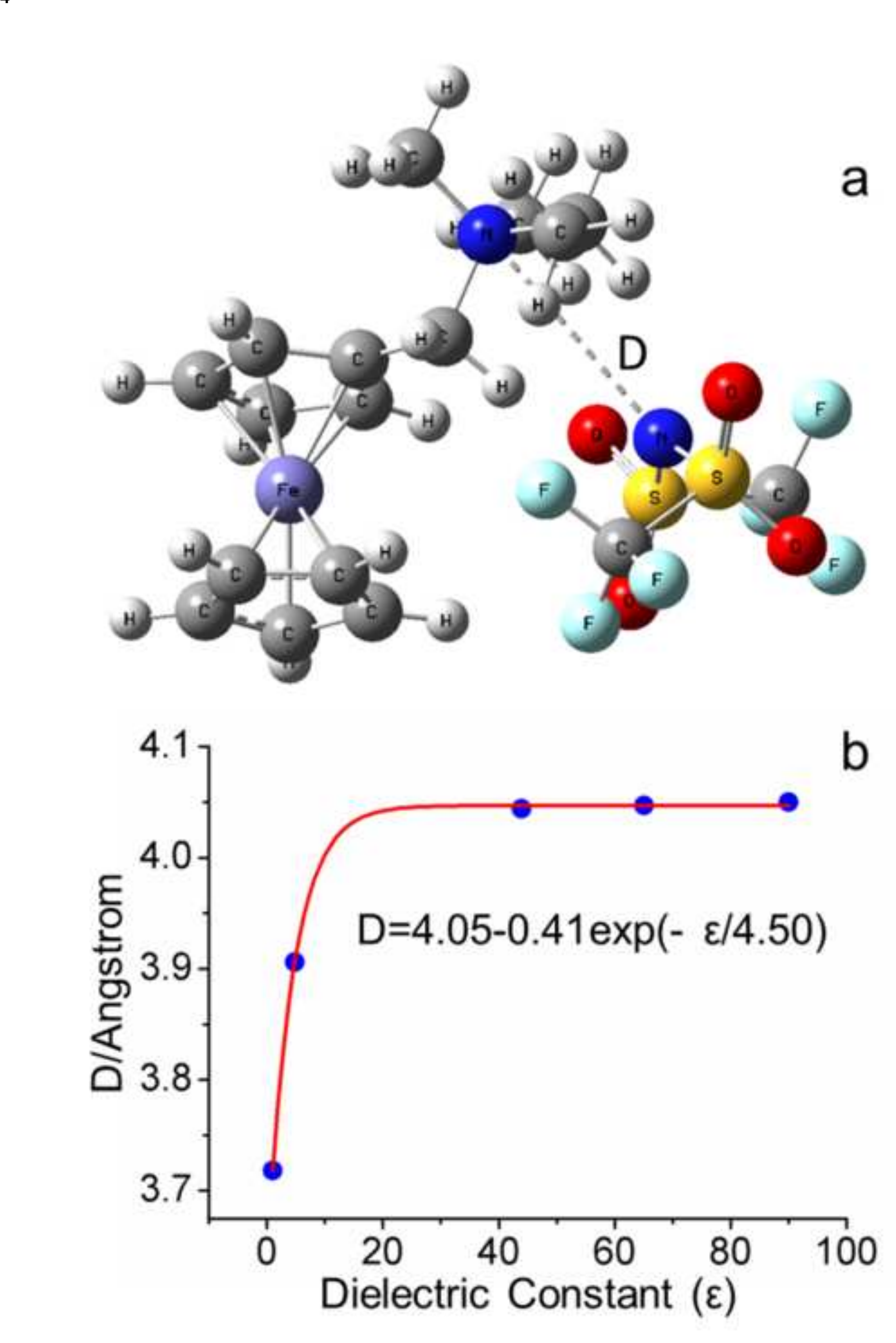

Figure 4

(⿸丆口

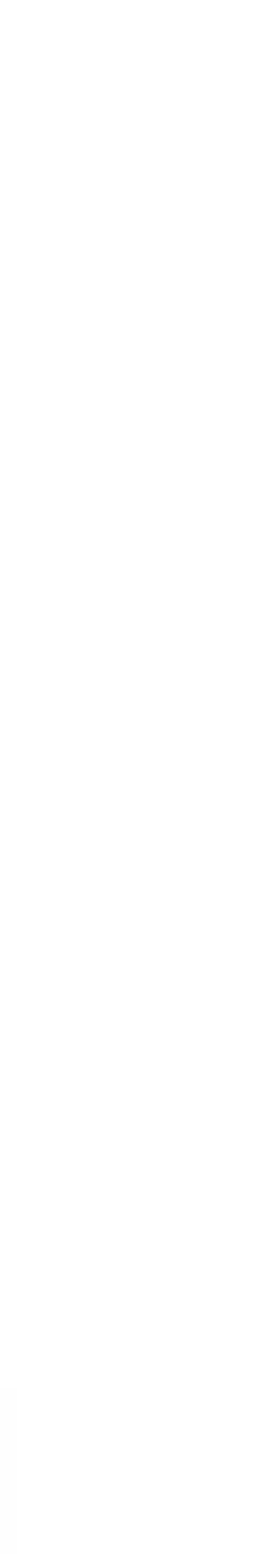

(

(

(
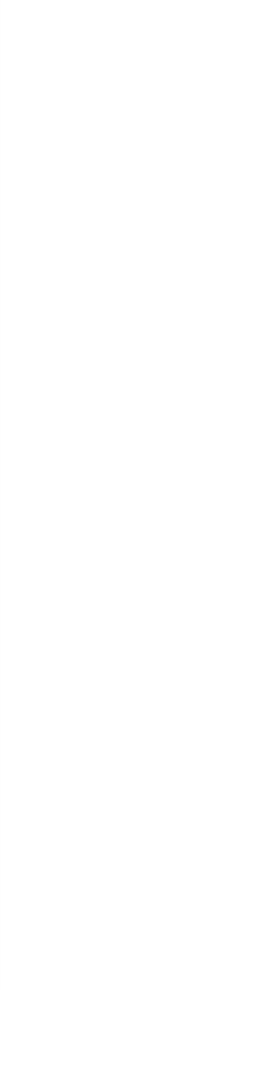

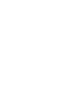



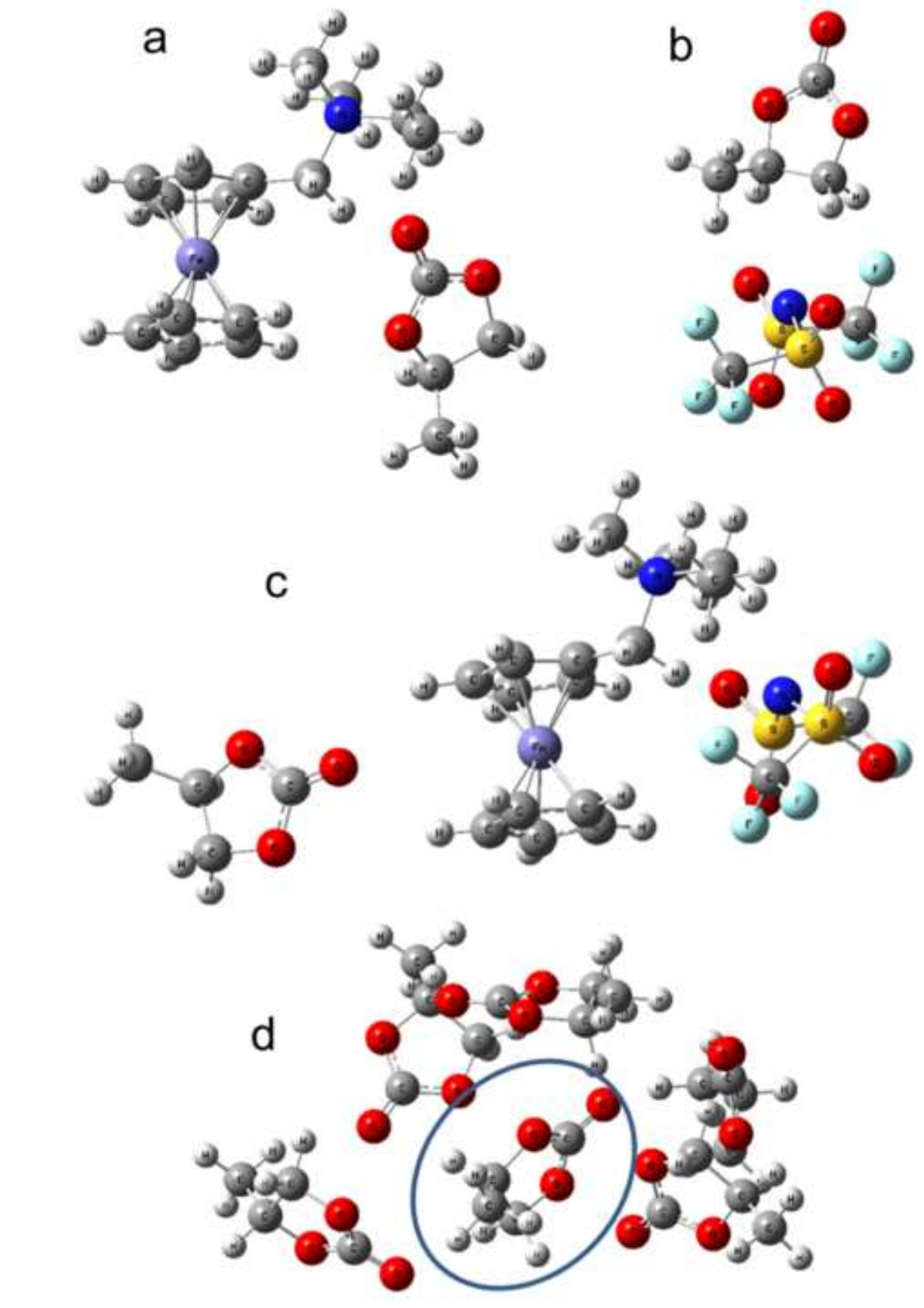

Figure 5

\section{Figure 5}

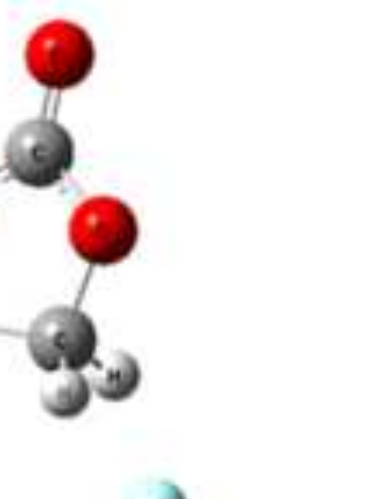




\section{Figure captions}

Chart 1. The labeling of the carbon sites and the formula of EC, PC, EMC Fc1N112 and TFSI .

Figure 1. The ${ }^{13} \mathrm{C}$ NMR spectra of (a) the solvent mixture of EC/PC/EMC (4/1/5 by weight); (b) $0.2 \mathrm{M} \mathrm{Fc;} \mathrm{(c)} 0.1 \mathrm{M}$ Fc1N112-TFSI; (d) <1.7 M Fc1N112-TFSI; (e) Saturated Fc1N112-TFSI.

Figure 2. ${ }^{17} \mathrm{O}$ NMR spectra of Fc1N112-TFSI dissolved in the solvent mixture of EC/PC/EMC at concentrations of (a) $0 \mathrm{M}$, (b) $0.25 \mathrm{M}$, (c) $0.85 \mathrm{M}$ and (d) $1.7 \mathrm{M}$.

Figure 3. Optimized structures of molecules and ions in the gas phase: (a) EC; (b) PC; (c) EMC; (d) Fc1N112+; (e) TFSI ${ }^{-}$.

Figure 4. The effect of $\varepsilon$ on structures of solute molecule. (a) The optimized structure of Fc1N112-TFSI in gas phase; (b) The relationship between D and $\varepsilon$.

Figure 5. Optimized structures of: (a) coordination of Fc1N112 and PC; (b) coordination of TFSI and PC; (c) coordination of Fc1N112-TFSI and PC; (d) six PC molecules, where one surrounded by five. Taking PC for example, EC and EMC are also calculated in these systems, and results are not shown in this figure. 
